\title{
Cranioplasty after decompressive craniectomy: is there a rationale for an initial artificial bone-substitute implant? A single-center experience after 631 procedures
}

\author{
Falko Schwarz, MD, ${ }^{1}$ Pedro Dünisch, MD, ${ }^{1}$ Jan Walter, MD, ${ }^{1}$ Yasser Sakr, PhD, MD, ${ }^{2}$ Rolf Kalff, MD, ${ }^{1}$ \\ and Christian Ewald, MD1
}

Departments of ${ }^{1}$ Neurosurgery and ${ }^{2}$ Anaesthesiology and Intensive Care, Jena University Hospital-Friedrich Schiller University
Jena, Germany

OBJECTIVE The complication rate for cranioplasty after decompressive craniectomy is higher than that after other neurosurgical procedures; aseptic bone resorption is the major long-term problem. Patients frequently need additional operations to remove necrotic bone and replace it with an artificial bone substitute. Initial implantation of a bone substitute may be an option for selected patients who are at risk for bone resorption, but this cohort has not yet been clearly defined. The authors' goals were to identify risk factors for aseptic bone flap necrosis and define which patients may benefit more from an initial bone-substitute implant than from autograft after craniectomy.

METHODS The authors retrospectively analyzed 631 cranioplasty procedures (503 with autograft, 128 with bone substitute) by using a stepwise multivariable logistic regression model and discrimination analysis.

RESULTS There was a significantly higher risk for reoperation after placement of autograft than after placement of bone substitute; aseptic bone necrosis $(n=108)$ was the major problem (OR 2.48 [95\% $\mathrm{Cl} 1.11-5.51])$. Fragmentation of the flap into 2 or more fragments, younger age (OR 0.97 [95\% Cl 0.95-0.98]; $p<0.001$ ), and shunt-dependent hydrocephalus (OR 1.73 [95\% Cl 1.02-2.92]; $p=0.04$ ) were independent risk factors for bone necrosis. According to discrimination analysis, patients younger than 30 years old and older patients with a fragmented flap had the highest risk of developing bone necrosis.

CONCLUSIONS Development of bone flap necrosis is the main concern in long-term follow-up after cranioplasty with autograft. Patients younger than 30 years old and older patients with a fragmented flap may be candidates for an initial artificial bone substitute rather than autograft.

http://thejns.org/doi/abs/10.3171/2015.4.JNS159

KEY WORDS bone substitute; autograft; cranioplasty; aseptic bone flap necrosis; bone resorption; trauma

$\mathrm{D}$ ECOMPRESSIVE craniectomy has become an inherent part of treatment for uncontrollable elevated intracranial pressure caused by various pathologies, including intracerebral bleeding and traumatic or ischemic brain swelling..$^{10,15,25,27}$ After stabilization of the neurological and medical condition of the patient, cranioplasty is generally performed for mechanical, cosmetic, and therapeutic reasons. $1,3,7-9,11-13,16,22,24,27,28$

The autologous cryoconserved or subcutaneously preserved bone flap has long been considered the gold standard for skull remodeling after craniectomy, ${ }^{6,14}$ but in recent years, a large number of short- and long-term complications after autologous bone flap reinsertion have been reported, including infection, bleeding, and aseptic bone resorption, which led to critical review of this technique. ${ }^{2,4,5,9,18,23,24}$ At the same time, the use of bone substitutes for cranioplasty has attracted interest. Although the cosmetic results of "hand-made" bone cement implants have been unsatisfactory, especially for large defects, new computer-assisted design implants with well-documented mechanical and esthetic properties are now available and provide a possible alternative to autograft cranioplasty. ${ }^{20}$

ABBREVIATIONS IQR = interquartile range; NPV = negative predictive value; $\mathrm{PEEK}=$ polyetheretherketone; $\mathrm{PPV}=$ positive predictive value; $\mathrm{SAH}=$ subarachnoid hemorrhage.

SUBMITTED January 4, 2015. ACCEPTED April 6, 2015.

INCLUDE WHEN CITING Published online September 25, 2015; DOI: 10.3171/2015.4.JNS159. 
With increasing concerns about the complications after autologous bone flap reinsertion, a new assessment of initial artificial bone-substitute implant placement is mandatory. The aim of our study, therefore, was to identify risk factors for aseptic bone flap necrosis and define which patients may benefit more from an initial bone-substitute implant than from an autograft after craniectomy.

\section{Methods}

This study was approved by the local ethics committee. Informed consent was not required because of the anonymous, observational, and retrospective character of the analysis.

\section{Data Acquisition}

All patients who had undergone cranioplasty after craniectomy at our institution between September 1994 and January 2014 were included. The patients' files and radiological examinations were screened retrospectively. We created a data set containing sex, age at the first operation, diagnosis, fragmentation, time between removal and replacement of the bone flap, type of graft, size of the implant, length of follow-up, presence of a CSF shunt, and surgically treated complications during follow-up. The diagnosis that led to the craniotomy was classified as diffuse brain injury, including traumatic brain swelling and traumatic intracerebral hematoma; brain swelling caused by subarachnoid hemorrhage (SAH), including vasospastic infarction; ischemic stroke (malignant middle cerebral artery infarction); tumor; other spontaneous intracerebral bleeding; other extraaxial hematoma, such as subacute or chronic subdural hematoma; infection; aseptic bone necrosis; or other.

The relative 2D size of the trepanation defect was calculated on a preoperative sagittal radiograph or a sagittal CT image by using the following formula: $A=\pi / 4 \times B \times$ $b$, where $\pi$ is a constant, and $B$ and $b$ are the largest diameters of an elliptic area, as described previously.9 The extent of possible aseptic bone necrosis was classified as 1 of 2 types according to CT features, as previously discussed (Fig. 1). ${ }^{9}$ Type I bone flap necrosis is a thinning of the flap, and Type II is defined as circumscribed, complete lysis of tabula interna and externa with loss of the bony protection of the brain. In our study, Type II bone flap necrosis
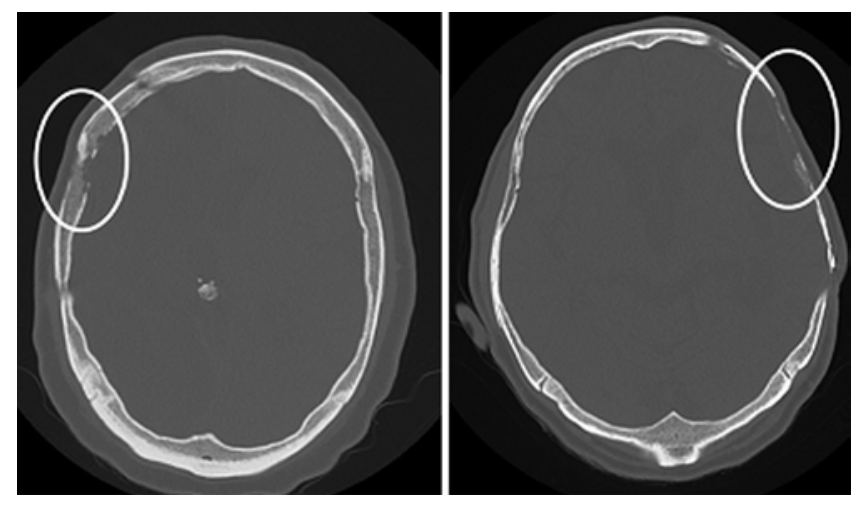

FIG. 1. CT images showing the types of bone flap resorption. Left: Type I, with thinning of the flap. Right: Type II, with circumscribed but complete lysis of tabula interna and externa. was considered an indication for surgical revision with resection of the necrotic bone and insertion of an artificial bone-substitute implant.

\section{Operative Procedure and Follow-Up}

The removed bone flap was cleaned, wrapped in sterile cloths, and stored at $-80^{\circ} \mathrm{C}$. Bone flaps fragmented by fracture or a former smaller craniotomy were reconstructed, when possible, with titanium plates (Biomet) before freezing. When required for insertion, the autologous bone flap was defrosted passively in sodium chloride solution at $37^{\circ} \mathrm{C}$. The skin incision was reopened, and the bony rims of the skull defect, including a dural layer, were prepared. The bone flap was reinserted and fixed with rigid titanium plates (Biomet). CSF was drained from the frontal horn if necessary. The dural layer was fixed to the bone flap with up to 5 central sutures in all cases, and 2 nonsuction drains were inserted below and above the bone flap. These drains remained in situ for 1-2 days. Antibiotic prophylaxis was administered as long as these drains were in place.

For artificial bone-substitute implants, a 1- to 2-mm axial thin-slice CT scan was performed to aid in creating an individually designed graft (3di $\mathrm{GmbH})$. The production of these ceramic, titanium, or polyetheretherketone (PEEK) implants takes at least 1-2 days. The operative technique to insert the implant was similar to that used for autograft insertion.

Follow-up was scheduled in our outpatient department according to an institutional protocol, with clinical and CT examinations 6 weeks, 3 months, 6 months, 12 months, and then every 12 months after bone graft implantation. For patients with a suspected or obvious complication, this follow-up regimen was adjusted depending on clinical and radiological findings.

\section{Statistical Analysis}

Data were analyzed by using the SPSS 17.0 for Windows software. Discrete variables are expressed as numbers (percentages) and continuous variables as the mean \pm standard deviation or median (interquartile ranges [IQRs]), unless stated otherwise. We established a stepwise multivariable logistic regression model with complications and Type II bone flap necrosis as dependent variables. The variables considered for this analysis were chosen according to previously published data ${ }^{9}$ and included age (per year), sex, primary neurosurgical diagnosis, time to reinsertion of the bone flap (months), number of fragments (categorized as 0,2, or $>2$ fragments), material (autograft or artificial bone-substitute implant), shunt-dependent hydrocephalus, and size of the bone flap (per $10 \mathrm{~cm}^{2}$ ). The Hosmer-Lemeshow test was used to assess the goodness of fit of the multivariable model. Covariates were retained in the model if the $p$ value was $<0.2$, and odds ratios with 95\% confidence interval values were computed.

Sensitivity, specificity, positive predictive values (PPVs), and negative predictive values (NPVs) were calculated for the variables found to be significantly associated with the risk of bone necrosis. For this analysis, we arbitrarily defined 3 age groups: $<30,31-60$, and $>60$ years.

All statistics were 2 -tailed, and any p value $<0.05$ was considered statistically significant. 


\section{Results}

\section{Patient Characteristics}

We included in our study 519 consecutive patients who underwent cranioplasty after craniectomy at our institution between September 1994 and January 2014. Four hundred sixty patients (mean age $49.5 \pm 18.3$ years; $59.8 \%$ male) received an autologous bone flap reinsertion; 158 $(34.3 \%)$ had diffuse traumatic brain injury, $81(17.6 \%)$ had cerebral infarction, 78 (16.9\%) had extraaxial bleeding, 74 $(16.1 \%)$ had subarachnoid hemorrhage, 52 (11.3\%) had intracerebral bleeding, and $17(3.7 \%)$ had a neoplasm. In 43 patients $(9.3 \%)$, bilateral bone flap implantation was necessary. Overall, 503 autologous bone flaps were reinserted.

One hundred twenty-four patients (mean age $43.5 \pm$ 20.4 years; $59.7 \%$ male) received an artificial bone-substitute implant; 4 patients needed a bilateral implant. A total of 128 implants were performed. The indication for the bone graft was aseptic necrosis of a previously inserted bone flap (58 implants in 54 patients) or infection of a replanted bone flap (8 after craniectomy, 16 after standard craniotomy). In 46 patients, there was no available autograft because of tumor infiltration, massive bone flap fragmentation, or infection; hence, in these patients a bone-substitute implant was inserted without failure of an initial autologous bone flap. The characteristics of this study group are listed in Table 1.

The median follow-up time was 11 months (IQR 2-23 months); 392 implants were followed for more than 6 months.

\section{Reoperation After Autologous Bone Flap Reinsertion}

During their hospital stay, 6 patients died as a result of pulmonary embolism $(n=3)$, myocardial infarction $(n=$ $1)$, or cerebral ischemia $(n=2)$. The 30 -day mortality rate was $1.3 \%$. A second surgical intervention was required during the hospital stay in 87 cases because of complications. In 31 (6.2\%) flaps, operative revision was necessary because of epidural, subdural, or intracerebral bleeding or subgaleal hematoma. Subduroperitoneal shunting was necessary in $21(4.2 \%)$ cases, wound debridement and/or flap explantation because of wound necrosis or deep infection in $33(6.6 \%)$ cases, and re-removal of the bone flap because of progressive brain swelling in $1(0.2 \%)$ case. Dislocation of the flap requiring refixation occurred in 4 patients during outpatient follow-up. Therefore, the overall short-term rate of complications that necessitated surgical revision $(n=87)$ or refixation of a loosened flap $(n=4)$ was $18.1 \%$.

\section{Aseptic Bone Necrosis}

On the last available CT images, surgically relevant resorption (Type II necrosis) of the bone was visible in 102 patients (mean age $47.9 \pm 18.9$ years; $57.7 \%$ male) and in $108(21.5 \%)$ bone flaps after a median time of 15 months (IQR 10-29 months) from reinsertion. Fifty-eight of these bone flaps were resected and replaced by a bone substitute. In the remaining 50 cases, the necrotic bone flap was not replaced because of refusal by the patient or his or her representative $(n=24)$, persistent vegetative neurological state $(n=20)$, or loss to follow-up after the initial CT scan $(n=6)$. Including the reoperation rate for aseptic bone ne-
TABLE 1. Graft characteristics*

\begin{tabular}{lcc}
\hline \multicolumn{1}{c}{ Graft Characteristic } & Autograft & Bone Substitute \\
\hline Type & & \\
\hline Bone & 503 & 128 \\
\hline Bioverit & - & 19 \\
\hline Titanium & - & 82 \\
\hline PEEK-Optima & - & 27 \\
\hline Time to insertion (days) & $82(59-117)$ & $370(158-654)$ \\
\hline Size & $86.2 \pm 22.6$ & $78.7 \pm 28.5$ \\
\hline$\leq 70 \mathrm{~cm}^{2}$ & $104(20.7)$ & $50(39.1)$ \\
\hline $71-90 \mathrm{~cm}^{2}$ & $183(36.4)$ & $30(23.4)$ \\
\hline $91-110 \mathrm{~cm}^{2}$ & $156(31)$ & $26(20.3)$ \\
\hline$>110 \mathrm{~cm}^{2}$ & $60(11.9)$ & $22(16.4)$ \\
\hline Fragmentation & & \\
\hline No fragments & $408(81.1)$ & 128 \\
\hline 2 fragments & $51(10.1)$ & - \\
\hline$>2$ fragments & $44(8.8)$ & - \\
\hline Follow-up CT (mos) & $11(2-23)$ & $14(5-39)$ \\
\hline
\end{tabular}

* The total number of patients was 631 . Values shown are number (\%), median $(\mathrm{IQR})$, or mean $\pm \mathrm{SD}$.

$\dagger$ Time to CT scan from which Type II bone flap necrosis was first diagnosed or time to last available CT, according to which no necrosis or Type I necrosis had occurred.

crosis, we had an overall reoperation rate of $29.6 \%$ (149 of 503) after cranioplasty using an autologous flap. Including Type II aseptic necrosis as a surgically relevant complication, the overall complication rate was 39.6\% (199 of 503).

\section{Reoperation After Artificial Bone-Substitute Implantation}

During the hospital stays, there were no deaths after bone-substitute insertion. In $9(7 \%)$ cases, surgical revision was necessary for epidural/subdural rebleeding. Subduroperitoneal shunting for persistent subdural hygroma was necessary in $5(3.9 \%)$ cases, and wound debridement because of wound necrosis or deep infection was necessary in $11(8.6 \%)$ cases. Dislocation of the implant that necessitated refixation occurred in 2 implants. The overall complication rate for operative revisions of the graft, therefore, was $21.1 \%$ (27 of 128).

\section{Autograft Versus Artificial Bone-Substitute Implant}

To assess the effect of the type of bone graft on the complication rate, we used 2 multivariable logistic regression models with "complication" as the dependent variable in the whole cohort $(n=519)$. In the first model, complication was defined as the need for surgical revision, including all Type II necrotic flaps (Table 2). The use of an autograft was associated with a significantly greater risk of developing a surgically relevant complication (OR 2.48 [95\% CI 1.11-5.51]; $\mathrm{p}=0.03$ ). When bone flap resorption was not considered a complication, the type of graft used was no longer significant, although there was a trend toward a lower risk of complications when an autograft was used. In this second model, a short time to replacement and fragmentation into 2 fragments were correlated with a lower risk of a surgically relevant complication. 
TABLE 2. Summary of multivariable logistic regression analyses with "surgically treated complication" as the dependent variable

\begin{tabular}{|c|c|c|c|c|}
\hline \multirow[b]{2}{*}{ Variable } & \multicolumn{2}{|c|}{ Including Bone Flap Resorption* } & \multicolumn{2}{|c|}{ Excluding Bone Flap Resorption† } \\
\hline & OR $(95 \% \mathrm{Cl})$ & $p$ Value & OR $(95 \% \mathrm{Cl})$ & p Value \\
\hline Age (per yr) & $0.99(0.98-1.00)$ & 0.012 & $1.01(1.00-1.03)$ & 0.14 \\
\hline Sex (female) & $0.91(0.62-1.35)$ & 0.63 & $0.6(0.35-1.05)$ & 0.07 \\
\hline \multicolumn{5}{|l|}{ Diagnosis } \\
\hline Diffuse brain injury & Reference & NA & Reference & NA \\
\hline $\mathrm{SAH}$ & $0.69(0.37-1.28)$ & 0.24 & $0.85(0.34-2.14)$ & 0.73 \\
\hline Cerebral infarction & $0.99(0.54-0.18)$ & 0.97 & $0.9(0.4-2.01)$ & 0.79 \\
\hline Tumor & $0.76(0.22-2.02)$ & 0.48 & $0.27(0.32-2.21)$ & 0.22 \\
\hline Intracerebral bleed & $0.88(0.44-1.76)$ & 0.72 & $0.91(0.36-2.34)$ & 0.85 \\
\hline Extraaxial bleed & $1.68(0.9-3.15)$ & 0.11 & $1.45(0.65-3.26)$ & 0.36 \\
\hline Time to replacement (mos) & $1.02(0.99-1.05)$ & 0.27 & $1.03(1.00-1.07)$ & 0.04 \\
\hline Material (autograft or bone substitute) & $2.48(1.11-5.51)$ & 0.03 & $0.48(0.21-1.11)$ & 0.08 \\
\hline Shunt-dependent hydrocephalus & $1.46(0.97-2.19)$ & 0.07 & $1.3(0.74-2.3)$ & 0.36 \\
\hline Size (per $10 \mathrm{~cm}^{2}$ ) & $1.02(0.82-1.25)$ & 0.89 & $1.18(0.88-1.57)$ & 0.28 \\
\hline \multicolumn{5}{|l|}{ Fragmentation } \\
\hline No fragments & Reference & NA & Reference & NA \\
\hline 2 fragments & $1.61(0.89-2.92)$ & 0.11 & $0.19(0.04-0.83)$ & 0.03 \\
\hline$>2$ fragments & $5.00(2.68-9.32)$ & $<0.001$ & $0.38(0.13-1.11)$ & 0.07 \\
\hline
\end{tabular}

\section{Risk Factors for Bone Flap Necrosis}

With aseptic necrosis identified as a significant factor for surgical revision, the next step was to define possible risk factors for bone resorption. A third multivariable logistic regression model, with "bone flap necrosis Type II" as the dependent variable (503 procedures), indicated that younger age (OR 0.97 [95\% CI 0.95-0.98]; p < 0.001), shunt-dependent hydrocephalus (OR 1.73 [95\% CI $1.02-2.92] ; \mathrm{p}=0.04$ ), and fragmentation into 2 (OR 3.71 [95\% CI 1.89-7.29]; $\mathrm{p}<0.001$ ) or more (OR 22.01 [95\% CI 9.43-51.61]; $\mathrm{p}<0.001$ ) fragments were significant risk factors for the development of aseptic bone flap necrosis (Table 3).

\section{Identifying Risk Groups for Bone Necrosis}

The final step was to define subgroups to help formulate clear indications for initial bone substitute rather than autograft. We arbitrarily created 14 subgroups considering age (0-30, 31-60, or 61-90 years), fragmentation (yes or no), and shunt-dependent hydrocephalus (yes or no) alone and in combination (Table 4). A high PPV for bone flap necrosis was found for all patients younger than 30 years (46.1\% without fragmentation, $80 \%$ with fragmentation, $66.7 \%$ with fragmentation and shunt) and in older patients with a fragmented bone flap without or with a shunt $(75.1 \%$ or $66.7 \%$, respectively).

\section{Discussion}

Removal of a bone flap during decompressive craniectomy results in the need for a second surgical intervention for skull remodeling. Once the neurological and medical states of the patient have been stabilized, bone grafting is performed for esthetic, but also therapeutic, reasons and is associated with postoperative neurological improvement. ${ }^{1,3,7-9,11-13,16,22,24,27}$

Compared with other neurosurgical interventions, the

TABLE 3. Summary of multivariable logistic regression analysis with "bone flap necrosis Type II" as the dependent variable*

\begin{tabular}{lcc}
\hline \multirow{2}{*}{ Variable } & \multicolumn{2}{c}{ Multivariable Analysis Results $\dagger$} \\
\cline { 2 - 3 } & OR $(95 \% \mathrm{Cl})$ & $\mathrm{p} \mathrm{Value}$ \\
\hline Age (per yr) & $0.97(0.95-0.98)$ & $<0.001$ \\
\hline Sex (female) & $1.34(0.81-2.23)$ & 0.26 \\
\hline Diagnosis & Reference & $\mathrm{NA}$ \\
\hline Diffuse brain injury & $0.77(0.35-1.67)$ & 0.51 \\
\hline SAH & $1.45(0.64-3.27)$ & 0.37 \\
\hline Cerebral infarction & $0.95(0.24-3.73)$ & 0.94 \\
\hline Tumor & $0.86(0.34-2.19)$ & 0.75 \\
\hline Intracerebral bleed & $1.76(0.74-4.1)$ & 0.2 \\
\hline Extraaxial bleed & $0.96(0.85-1.1)$ & 0.49 \\
\hline Time to replacement (mos) & $1.73(1.02-2.92)$ & 0.04 \\
\hline Shunt-dependent hydrocephalus & $0.88(0.67-1.17)$ & 0.39 \\
\hline Size (per $\left.10 \mathrm{~cm}^{2}\right)$ & & \\
\hline Fragmentation & Reference & $\mathrm{NA}$ \\
\hline No fragments & $3.71(1.89-7.29)$ & $<0.001$ \\
\hline 2 fragments & $22.01(9.43-51.61)$ & $<0.001$ \\
\hline$>2$ fragments & & \\
\hline
\end{tabular}

* A total of 503 procedures were considered.

$\dagger$ Hosmer-Lemeshow chi-square $=6.44 ; p=0.599 ;$ Nagelkerke $R^{2}=0.306$. 
TABLE 4. PPV, NPV, sensitivity, and specificity for bone flap necrosis in 503 procedures

\begin{tabular}{ccccc}
\hline \multicolumn{1}{c}{ Variable } & $\begin{array}{c}\text { PPV } \\
(\%)\end{array}$ & $\begin{array}{c}\text { NPV } \\
(\%)\end{array}$ & $\begin{array}{c}\text { Sensitivity } \\
(\%)\end{array}$ & $\begin{array}{c}\text { Specificity } \\
(\%)\end{array}$ \\
\hline Age & & & & \\
\hline 0-30 yrs & 46.1 & 82.5 & 40.2 & 85.8 \\
\hline Fragmentation & 80 & 77.3 & 12.6 & 99.0 \\
\hline Shunt & 48.7 & 78.9 & 16.2 & 94.8 \\
\hline Shunt \& fragmentation & 66.7 & 77.3 & 3.4 & 99.5 \\
\hline 31-60 yrs & 18.7 & 71.8 & 41.9 & 44.8 \\
\hline Fragmentation & 75.1 & 78.4 & 10.3 & 99 \\
\hline Shunt & 25.5 & 77.3 & 23.1 & 79.5 \\
\hline Shunt \& fragmentation & 66.7 & 77 & 1.7 & 99.7 \\
\hline 61-90 yrs & 15.1 & 73.6 & 17.9 & 69.4 \\
\hline Fragmentation & NA & NA & NA & NA \\
\hline Shunt & NA & NA & NA & NA \\
\hline Shunt \& fragmentation & NA & NA & NA & NA \\
\hline Fragmentation & 18 & 20.9 & 70.9 & 2.3 \\
\hline Shunt & 20.2 & 71.6 & 54.7 & 34.5 \\
\hline
\end{tabular}

$\mathrm{NA}=$ not available because of the small number of events.

complication rate of cranioplasty after decompressive craniectomy is surprisingly high, with a reoperation rate for wound infection, rebleeding, and wound-healing problems of up to $36.5 \% .^{23,26}$ Long-term complications after autograft implantation have become an increasing concern in recent years. Aseptic resorption of the reimplanted flap is considered a particular problem, having been described in up to $50 \%$ of patients, and although age, fragmentation, and shunt dependency have been postulated as possible risk factors, the causes remain unclear. ${ }^{2-5,9,18,23,24}$

Another possible influence on bone flap necrosis is storage of the bone flaps. Some authors have reported a higher incidence of aseptic bone resorption with cryoconserved flaps than with bone flaps stored in abdominal subcutaneuous fat. ${ }^{2}$ A disadvantage of abdominal storage is the second operative site, and some authors have also described a lysis of the subcutaneous implanted flap by macrophages. Therefore, retention in a freezer is the preference in our clinic.

New computer-assisted design implants made of titanium, ceramics, or PEEK represent a possible alternative to autograft cranioplasty; biomechanical properties and excellent cosmetic results after insertion have been well documented. ${ }^{17,19-21}$ However, because of their high costs compared with autologous flaps and a suspected higher complication rate for allogenic materials, these materials are not widely used for initial cranioplasty.

Against this background-high reoperation rate after autologous bone flap reinsertion on the one hand and effective new materials and techniques on the other-we analyzed more than 600 procedures to define for the first time a risk profile for different patient subgroups in terms of risks of reoperation and to formulate a study-based recommendation for initial autograft or artificial bone-substitute implantation in each patient subgroup.

We first examined the reoperation rates after autograft and bone-substitute insertion and found a significantly higher risk for reoperation after cranioplasty using the autologous flap. Then, excluding aseptic bone resorption as a complication, we found that the risk profile for reoperation was comparable in the 2 groups, thus identifying aseptic bone necrosis as the crucial factor for the higher reoperation rate after autograft over that after bone-substitute implantation. Younger age, bone flap fragmentation, and shunt-dependent hydrocephalus were independent risk factors for bone resorption. Although the impact of bone flap fragmentation on bone necrosis is clear, the effects of age and hydrocephalus are less obvious, although some recent studies made similar findings. ${ }^{3,9,24}$ Other variables, such as initial diagnosis, size of the flap, and time to reinsertion, did not seem to affect the risk of reoperation.

Therefore, there may be a rationale for initial artificial bone-substitute implantation in some patients after cranioplasty. Our data provide a basis for an individually adjusted therapeutic approach based on risk factors for bone resorption (i.e., younger age, bone flap fragmentation, and shunt dependency). On the basis of PPV, NPV, sensitivity, and specificity results, we recommend that an initial bonesubstitute implant be considered for all patients younger than 30 years irrespective of the presence of fragmentation or shunting and in older patients (30-60 years old) when the bone flap is fragmented. For patients older than 60 years, the number of events (bone flap necrosis and fragmentation) was too low to make a definite conclusion.

Our study had some limitations. First, the single-center retrospective design is a clear drawback. In our cohort, 6 patients were lost to follow-up, and only 392 patients were followed for more than 6 months after the cranioplasty, although the rate of aseptic bone necrosis would be expected to be higher with longer follow-up periods. Second, the observational nature of the study did not allow for a comparison between autograft and bone substitute in a randomized manner, and the groups varied in numbers of patients and time to replacement. Nonetheless, our results are hypothesis generating, and the aim of our analysis was not to compare between outcomes according to the type of bone flaps but to identify patients at increased risk of complications from autograft. These patients should be ideally suited for primary artificial bone-substitute implants. The results of our study, therefore, are crucial and should be considered in the design of future studies on the subject. Third, the age groups were chosen somewhat arbitrarily. Another question to be answered in the future is the possible advantage of one bone-substitute material over another. The results of some studies suggested that PEEK may be better than titanium..$^{17}$ In our cohort, the groups were too small to make valid comparisons.

\section{Conclusions}

Bone flap resorption is a key complication after cranioplasty using autologous bone flaps. Younger age, shunt dependency, and bone flap fragmentation are independent risk factors for reoperation. We therefore suggest that an initial artificial bone-substitute implant rather than autograft be considered in patients younger than 30 years and in all patients with a fragmented flap. In addition, 
prospective evaluation, including a robust definition of relevant bone flap necrosis and clearly defined clinical and radiological follow-up, is necessary to confirm these recommendations. A randomized trial comparing these 2 cranioplasty media (initial autograft vs initial bone substitute) would be a useful tool for providing more evidence.

\section{References}

1. Archavlis E, Carvi Y Nievas M: The impact of timing of cranioplasty in patients with large cranial defects after decompressive hemicraniectomy. Acta Neurochir (Wien) 154:1055-1062, 2012

2. Beauchamp KM, Kashuk J, Moore EE, Bolles G, Rabb C, Seinfeld J, et al: Cranioplasty after postinjury decompressive craniectomy: is timing of the essence? J Trauma 69:270274, 2010

3. Bowers CA, Riva-Cambrin J, Hertzler DA II, Walker ML: Risk factors and rates of bone flap resorption in pediatric patients after decompressive craniectomy for traumatic brain injury. J Neurosurg Pediatr 11:526-532, 2013

4. Carvi Y Nievas MN, Höllerhage HG: Early combined cranioplasty and programmable shunt in patients with skull bone defects and CSF-circulation disorders. Neurol Res 28:139144, 2006

5. Chang V, Hartzfeld P, Langlois M, Mahmood A, Seyfried D: Outcomes of cranial repair after craniectomy. J Neurosurg 112:1120-1124, 2010

6. Cheng CH, Lee HC, Chen CC, Cho DY, Lin HL: Cryopreservation versus subcutaneous preservation of autologous bone flaps for cranioplasty: comparison of the surgical site infection and bone resorption rates. Clin Neurol Neurosurg 124:85-89, 2014

7. Chieregato A: The syndrome of the sunken skin flap: a neglected potentially reversible phenomenon affecting recovery after decompressive craniotomy. Intensive Care Med 32:1668-1669, 2006

8. Dujovny M, Fernandez P, Alperin N, Betz W, Misra M, Mafee M: Post-cranioplasty cerebrospinal fluid hydrodynamic changes: magnetic resonance imaging quantitative analysis. Neurol Res 19:311-316, 1997

9. Dünisch P, Walter J, Sakr Y, Kalff R, Waschke A, Ewald C: Risk factors of aseptic bone resorption: a study after autologous bone flap reinsertion due to decompressive craniotomy. J Neurosurg 118:1141-1147, 2013

10. El Ahmadieh TY, Adel JG, El Tecle NE, Daou MR, Aoun SG, Nanney AD III, et al: Surgical treatment of elevated intracranial pressure: decompressive craniectomy and intracranial pressure monitoring. Neurosurg Clin N Am 24:375391, 2013

11. Ewald C, Duenisch P, Walter J, Götz T, Witte OW, Kalff R, et al: Bone flap necrosis after decompressive hemicraniectomy for malignant middle cerebral artery infarction. Neurocrit Care 20:91-97, 2014

12. Gooch MR, Gin GE, Kenning TJ, German JW: Complications of cranioplasty following decompressive craniectomy: analysis of 62 cases. Neurosurg Focus 26(6):E9, 2009

13. Grant GA, Jolley M, Ellenbogen RG, Roberts TS, Gruss JR, Loeser JD: Failure of autologous bone-assisted cranioplasty following decompressive craniectomy in children and adolescents. J Neurosurg 100 (2 Suppl Pediatrics):163-168, 2004

14. Inamasu J, Kuramae T, Nakatsukasa M: Does difference in the storage method of bone flaps after decompressive craniectomy affect the incidence of surgical site infection after cranioplasty? Comparison between subcutaneous pocket and cryopreservation. J Trauma 68:183-187, 2010

15. Jasielski P, Głowacki M, Czernicki Z: Decompressive craniectomy in trauma: when to perform, what can be achieved. Acta Neurochir Suppl 118:125-128, 2013
16. Kemmling A, Duning T, Lemcke L, Niederstadt T, Minnerup $\mathrm{J}$, Wersching $\mathrm{H}$, et al: Case report of MR perfusion imaging in sinking skin flap syndrome: growing evidence for hemodynamic impairment. BMC Neurol 10:80, 2010

17. Lethaus B, Safi Y, ter Laak-Poort M, Kloss-Brandstätter A, Banki F, Robbenmenke C, et al: Cranioplasty with customized titanium and PEEK implants in a mechanical stress model. J Neurotrauma 29:1077-1083, 2012

18. Liang W, Xiaofeng Y, Weiguo L, Gang S, Xuesheng Z, Fei $\mathrm{C}$, et al: Cranioplasty of large cranial defect at an early stage after decompressive craniectomy performed for severe head trauma. J Craniofac Surg 18:526-532, 2007

19. Miyake H, Ohta T, Tanaka H: A new technique for cranioplasty with L-shaped titanium plates and combination ceramic implants composed of hydroxyapatite and tricalcium phosphate (Ceratite). Neurosurgery 46:414-418, 2000

20. Ng ZY, Nawaz I: Computer-designed PEEK implants: a peek into the future of cranioplasty? J Craniofac Surg 25:e55e58, 2014

21. Okumura T, Oda Y, Mori K, Uchida Y, Morimoto M, Kamimura Y, et al: [Alumina ceramic (Bioceram) as the cranioplastic material-experimental study and application in cranioplasty.] No Shinkei Geka 12 (3 Suppl):246-252, 1984 (Jpn)

22. Schorl M: Sinking skin flap syndrome (SSFS) _ clinical spectrum and impact on rehabilitation. Cent Eur Neurosurg 70:68-72, 2009

23. Schuss P, Vatter H, Marquardt G, Imöhl L, Ulrich CT, Seifert V, et al: Cranioplasty after decompressive craniectomy: the effect of timing on postoperative complications. J Neurotrauma 29:1090-1095, 2012

24. Schuss P, Vatter H, Oszvald A, Marquardt G, Imöhl L, Seifert $\mathrm{V}$, et al: Bone flap resorption: risk factors for the development of a long-term complication following cranioplasty after decompressive craniectomy. J Neurotrauma 30:91-95, 2013

25. Scotter J, Iorga R, Stefanou D, Wilson MH: Management of malignant middle cerebral artery infarction following a cardiac stab wound--the role of early decompressive craniectomy. Br J Neurosurg 28:534-535, 2014

26. Sobani ZA, Shamim MS, Zafar SN, Qadeer M, Bilal N, Murtaza SG, et al: Cranioplasty after decompressive craniectomy: An institutional audit and analysis of factors related to complications. Surg Neurol Int 2:123, 2011

27. Takeuchi S, Takasato Y, Masaoka H, Hayakawa T, Yatsushige $\mathrm{H}$, Shigeta K, et al: Decompressive craniectomy with hematoma evacuation for large hemispheric hypertensive intracerebral hemorrhage. Acta Neurochir Suppl 118:277-279, 2013

28. Yamashima T: Cranioplasty with hydroxylapatite ceramic plates that can easily be trimmed during surgery. A preliminary report. Acta Neurochir (Wien) 96:149-153, 1989

\section{Disclosure}

The authors report no conflict of interest concerning the materials or methods used in this study or the findings specified in this paper.

\section{Author Contributions}

Conception and design: Ewald. Acquisition of data: Schwarz, Dünisch, Walter, Ewald. Analysis and interpretation of data: Ewald. Critically revising the article: Sakr, Ewald. Reviewed submitted version of manuscript: Sakr, Kalff, Ewald. Statistical analysis: Sakr. Study supervision: Kalff, Ewald.

\section{Correspondence}

Falko Schwarz, Department of Neurosurgery, Jena University Hospital-Friedrich Schiller University Jena, Erlanger Allee 101, Jena 07747, Germany. email: falko.schwarz@med.uni-jena.de. 